\title{
The safety of sulfonylurea therapy in type 2 diabetes: have we reached the practical limits of our evidence base?
}

\author{
Jeffrey A. Johnson
}

Received: 8 September 2014 / Accepted: 30 September 2014 / Published online: 17 October 2014

(C) Springer-Verlag Berlin Heidelberg 2014

Keywords Cardiotoxicity · Epidemiology · Hypoglycaemia - Observational studies · Safety . Sulfonylureas

\section{Abbreviations \\ GRADE Glycemia Reduction Approaches in Diabetes: A Comparative Effectiveness Study \\ UGDP University Group Diabetes Project \\ UKPDS UK Prospective Diabetes Study}

Few clinical controversies have persisted as long as that of the safety of sulfonylurea drugs in the management of type 2 diabetes mellitus. It has been nearly 45 years since the report from the University Group Diabetes Project (UGDP) of a significantly higher rate of cardiovascular-related death in patients using tolbutamide compared with placebo [1]. The subsequent and much larger UK Prospective Diabetes Study (UKPDS) did not confirm those findings, and in fact suggested intensified therapy with glibenclamide was associated with reduced microvascular complications in type 2 diabetes compared with conventional therapy [2]. Thus, sulfonylureas have maintained a strong position among the growing treatment options, also in large measure owing to their low cost relative to newer therapeutic agents arriving on the market.

Nonetheless, numerous reports, largely using observational study designs, have continued to explore the potential negative consequences of sulfonylureas, often attempting to discern clinical differences amongst the numerous sulfonylurea compounds that have entered the market since the UGDP and

J. A. Johnson $(\bowtie)$

School of Public Health, University of Alberta, 2040 Li Ka Shing

Center, Edmonton T6G 2E3, Canada

e-mail: jeff.johnson@ualberta.ca
UKPDS, with the assumed benefit of an improved safety profile [3]. Of primary concern are the risks of hypoglycaemia and potential cardiotoxicity. Differences in the risk of these untoward effects are thought to be due to their metabolic clearance and the potency and selectivity of the various agents for sulfonylurea receptors on the pancreas and the heart [4].

In this issue of Diabetologia, Mogensen and colleagues contribute a new observational study on this topic, using population-based data from Denmark to compare the risk of all-cause mortality, cardiovascular events and hypoglycaemia in patients using combinations of sulfonylurea and insulin or metformin and insulin [5]. Among the approximately 25,000 individuals (median treatment duration 3 years) there were over 1560 deaths, of which nearly 600 were from cardiovascular causes. After adjusting for potential confounding variables available in the linkable databases, the authors observed an $81 \%$ increase in overall mortality and $35 \%$ increased risk of cardiovascular death amongst those on sulfonylurea plus insulin compared with metformin plus insulin. Not surprisingly, the combination of sulfonylurea plus insulin more than doubled the risk of hypoglycaemia compared with the metformininsulin combination [5]. No differences were observed for any of the outcomes when the authors compared the various sulfonylureas used in combination with insulin.

Like similar epidemiological studies that have used large population databases, Mogensen and colleagues were able to efficiently assemble large cohorts with a sizable number of total events over a long period of time [5]. Nonetheless, they undoubtedly had limited power to compare clinical events between the various sulfonylureas. Also in common with similar epidemiological studies, Mogensen and colleagues were only able to adjust for differences between treatment groups for variables that could be derived from the administrative databases. Thus, as the authors themselves have appropriately noted, baseline differences existed between groups, and residual confounding is a possible alternative explanation, 
limiting the interpretation of the observed associations. Given the considerable focus on the potential cardiotoxicity of sulfonylureas [5], even if mediated through hypoglycaemia, one wonders why the elevated risk of all-cause mortality is greater than the elevated risk of cardiovascular-related death compared with metformin. This suggests that sulfonylureas have other, noncardiovascular adverse effects or, alternatively, metformin has other non-cardiovascular benefits.

Pharmacoepidemiological studies of glucose-lowering drugs used in the management of type 2 diabetes are typically challenged by the concern of channelling or confounding by indication. Like other similar studies, this may be at play in the report by Mogensen et al, whereby patients being treated with sulfonylureas plus insulin were clinically different from those treated with metformin plus insulin, and these clinical differences, which may also be related to the outcomes of all-cause or cardiovascular death, are not fully accounted for in the statistical adjustments undertaken with the multivariate analysis (i.e. residual confounding). Typically, important clinical measures such as blood pressure, lipids, renal function, weight or smoking status are not available for comparison or adjustment. Instead, proxy measures of drug therapy or diagnostic codes from billing claims are used to characterise comorbidities. Methodologically stronger pharmacoepidemiological studies in the future will need to involve the linkage of large clinical databases with population-based administrative claims databases.

As a consequence of the progressive nature of dysglycaemia, treatment regimens change over time, in terms of both the agents used and the dosages prescribed. Mogensen and colleagues generally applied strong and accepted practices in their methods, including accounting for time-varying exposure in their regression models, a propensity score matched comparison and various sensitivity analyses for different exposure definitions. Nonetheless, one wonders whether the complete exposure history to all glucose-lowering therapy was fully accounted for, given that the timescale for the comparisons began with the first claimed co-prescription for insulin and sulfonylurea or metformin.

Another important consideration is the clinical relevance of the comparison undertaken in this latest Danish study. The main comparison is between the combinations of sulfonylurea plus insulin and metformin plus insulin. As the authors themselves point out, the former is not a recommended or common combination for the treatment of type 2 diabetes [5]. In the Danish diabetic population it was clearly a diminishing strategy [5], in part because of the growing predominance of metformin, but also to the well-recognised risk of hypoglycaemia. Insulin is, after all, the most potent hypoglycaemic agent, so combination with oral agents also known to induce hypoglycaemia is unlikely to be a leading clinical strategy. Indeed, more relevant comparisons would be different second-line therapies added to metformin therapy.

To address this important clinical question, the US National Institute of Diabetes and Digestive and Kidney Diseases (NIDDK) is financially supporting the Glycemia Reduction Approaches in Diabetes: A Comparative Effectiveness Study (GRADE) [6]. GRADE is a controlled trial enrolling 5,000 metformin-treated patients with type 2 diabetes, randomising in an unmasked fashion to medications from four different classes (sulfonylureas, dipeptidyl peptidase 4 inhibitors, glucagon-like peptide 1 receptor agonists and insulin). With a planned follow-up of nearly 5 years, the main outcomes are glycaemic control and the need to go on to basal insulin therapy, with secondary outcomes of microvascular complications and cardiovascular risk; importantly, quality of life and cost-effectiveness of the various combination therapies will also be assessed in this comparative effectiveness trial [6]. While GRADE will be a powerful and important study, like any study, it will inevitably be limited in its ability to answer other important and relevant clinical questions. New drugs entering the market for the treatment of type 2 diabetes need to demonstrate benefits in reducing cardiovascular events; GRADE will only assess cardiovascular risk factors. Moreover, combination therapy with the most recently introduced treatment options, the sodium-glucose co-transporter 2 (SGLT2) receptor inhibitors [7], which are increasingly being used in several markets, will not be addressed by GRADE.

Some years ago, Tonelli wrote about the philosophical limits of evidence-based medicine, noting that there is an 'intrinsic gap between clinical research and clinical practice' [8]. He suggested that evidence-based medicine essentially devalues the individuality of the patient and shifts the focus of clinical practice away from caring for individuals towards the care of populations. Given the limitations inherent in even the most well-designed and implemented observational studies or randomised controlled trials, one wonders if we might also consider the practical limits of evidence-based medicine. This might be particularly true for the choice of sulfonylurea therapy for type 2 diabetes, among the other potential choices to be added to first-line metformin therapy. The current evidence base for differences in outcomes between sulfonylurea agents is conflicting. Balancing their known risks of hypoglycaemia, the pharmacological differences between agents and their very low costs, it still remains up to the individual clinician working with the individual patient to make the best therapeutic choice for them.

Contribution statement JAJ was the sole contributor to this paper.

Duality of interest The author declares that there is no duality of interest associated with this manuscript. 


\section{References}

1. Meinert CL, Knatterud GL, Prout TE, Klimt CR (1970) A study of the effects of hypoglycemic agents on vascular complications in patients with adult-onset diabetes. II. Mortality results. Diabetes 19(Suppl): 789-830

2. UKPDS Group (1998) Intensive blood-glucose control with sulphonylureas or insulin compared with conventional treatment and risk of complications in patients with type 2 diabetes (UKPDS 33). UK Prospective Diabetes Study (UKPDS) Group. Lancet 352:837853

3. Schwartz TB, Meinert CL (2004) The UGDP controversy: thirty-four years of contentious ambiguity laid to rest. Perspect Biol Med 47:564 574
4. Abdelmoneim AS, Hasenbank SE, Seubert JM, Brocks DR, Light PE, Simpson SH (2012) Variations in tissue selectivity amongst insulin secretagogues: a systematic review. Diabetes Obes Metab 14:130-138

5. Mogensen UM, Andersson C, Fosbøl EL et al (2014) Sulfonylurea in combination with insulin is associated with increased mortality compared with a combination of insulin and metformin in a retrospective Danish nationwide study. Diabetologia. doi:10.1007/s00125-0143372-z

6. Nathan DM, Buse JB, Kahn SE et al (2013) Rationale and design of the Glycemia Reduction Approaches in Diabetes: A Comparative Effectiveness Study (GRADE). Diabetes Care 36:2254-2261

7. Hasan FM, Alsahli M, Gerich JE (2014) SGLT2 inhibitors in the treatment of type 2 diabetes. Diabetes Res Clin Pract 104:297-322

8. Tonelli MR (1998) The philosophical limits of evidence-based medicine. Acad Med 73:1234-1240 